\title{
Can Weed Hosts Increase Aggressiveness of Phytophthora infestans on Potato?
}

\author{
L. Grönberg, B. Andersson, and J. Yuen
}

Department of Forest Mycology and Plant Pathology, Swedish University of Agricultural Sciences, P.O. Box 7026 , SE-750 07 Uppsala, Sweden.

Accepted for publication 8 December 2011.

\section{ABSTRACT}

Grönberg, L., Andersson, B., and Yuen, J. 2012. Can weed hosts increase aggressiveness of Phytophthora infestans on potato? Phytopathology 102:429-433.

Potato late blight, caused by Phytophthora infestans, is a major disease in potato production throughout the world. In southern Sweden, hairy nightshade (Solanum physalifolium), an alternative non-crop host to the pathogen, is an increasing weed problem. Single-lesion leaves infected by $P$. infestans were collected from potato and hairy nightshade to determine phenotypic and genotypic population differentiation of $P$. infestans between the two hosts. Genotypic variation was estimated using micro- satellites as markers. The results showed no genotypic differentiation in the samples between the two hosts. Aggressiveness tests were performed using the sampled isolates to cross-inoculate potato and hairy nightshade. The proportion of infected leaves, latency period, lesion growth rate, and sporulation capacity were measured. For isolates from hairy nightshade, the odds of infection were higher on both hosts combined. When tested on potato leaves, isolates from hairy nightshade showed a significantly shorter latency period and higher sporulation capacity compared with isolates from potato. This indicates that an alternative host can filter populations of $P$. infestans toward a higher aggressiveness, which could lead to increasing problems in controlling potato late blight.
The oomycete Phytophthora infestans is the causal agent of potato late blight, a major disease in potato throughout the world (18). The $P$. infestans population in the Nordic region differs from what has been reported from other countries. In the Nordic region, the two mating types of the pathogen are evenly distributed, oospore formation is common in infected potato crops, and there are strong indications of primary infections caused by oospores. This points to a substantial impact of sexual reproduction $(2,3$, 32 ). As a result, the genotypic variation of populations of $P$. infestans is high compared with most other potato-producing regions.

The host range of $P$. infestans is mainly restricted to the family Solanaceae, of which potato (Solanum tuberosum) and tomato $(S$. lycopersicum) are the most important agricultural crops (12). In Sweden, there are a few wild Solanum spp. that are reported as hosts for $P$. infestans. The most widespread are $S$. nigrum (black nightshade, a common weed in potato crops), and $S$. dulcamara (bittersweet). Both these species can be infected by $P$. infestans under field conditions in Europe $(9,13,19)$. However, attacks by $P$. infestans on $S$. dulcamara and $S$. nigrum are very rare and must be considered to have no or very limited effect on late blight epidemics $(7,13)$. During the last 30 years, another Solanum sp., S. physalifolium Rusby var. nitidibaccatum (Bitter) Edmonds (10) (in this article, referred to as $S$. physalifolium or hairy nightshade), has established itself as a serious weed in southern Sweden. Hairy nightshade is a summer annual plant native to South America, but it has been widely introduced elsewhere in the world. Problems with hairy nightshade as a weed occur mostly in row crops with low competitive ability such as potato, sugar beet, and carrot (10). These crops are also characterized by late planting, which favors nightshades that require a relatively high germi-

Corresponding author: L. Grönberg; E-mail address: lina.gronberg@ @lu.se

http://dx.doi.org/10.1094/PHYTO-07-11-0192

(c) 2012 The American Phytopathological Society nation temperature. Also, hairy nightshade, with its shallow root system, can benefit from irrigation, which has become a common practice during recent decades in Sweden.

Unlike black nightshade and bittersweet, hairy nightshade is highly susceptible to $P$. infestans in Sweden, and attacks are observed every year. Studies in the field and under laboratory conditions have shown that $P$. infestans infections on $S$. physalifolium can sustain production of zoosporangia and oospores (1). As a result, this alternative host plant might affect the spread of $P$. infestans during the growing season as well as between seasons. Thus far, S. physalifolium has mainly been restricted to the southernmost parts of Sweden but, with the predicted increases in temperature due to climate change, it could also establish itself as a weed problem further north. Interestingly enough, there are no reports of $P$. infestans infections on $S$. physalifolium under field conditions outside Sweden. However, it is important to be aware of the taxonomical uncertainty within the Solanaceae family. Edmonds (10) specifically mentions that $S$. physalifolium is often misidentified as $S$. sarrachoides, which is reported as a host for $P$. infestans in North America (9). In addition, hairy nightshade is used as the trivial name for both these species. Naturally occurring hybrids between different species of Solanum also add to the unclear taxonomic status (10).

In one study comparing $P$. infestans from tomato and potato (28), it was found that isolates were more aggressive on their respective source host (measured in terms of lesion size) than on their alternative host. One general definition of aggressiveness is the quantity of disease caused by a pathogen on a compatible host (31). In the interaction between pathogen and host, there are several factors that influence the amount of disease: the environment (e.g., humidity, temperature, and light), the traits of the pathogen (e.g., spore production and growth rate), and the traits of the host (e.g., major and minor gene resistance, morphology, and age). When assessing aggressiveness, variations in pathogen-dependent traits are usually determined while keeping environmental and host factors constant. Aggressiveness of $P$. infestans in potato or tomato has been extensively studied $(4,8,14,22)$. How- 
ever, less work has been done on aggressiveness of this pathogen on non-crop Solanaceae species $(15,27,30)$.

The study presented here was done to investigate whether different hosts-in this case, S. physalifolium and S. tuberosumwill lead to phenotypic or genotypic population differentiation in $P$. infestans. Molecular markers (simple-sequence repeats [SSRs]) were used to assess the genotypic differentiation, while phenotypic differentiation was evaluated by aggressiveness tests. Aggressiveness was measured via different components: the odds of infection (proportion of infected leaves), latency period (time between inoculation and sporulation), lesion growth (rate of lesion expansion per day), and sporulation capacity (number of sporangia formed per lesion).

\section{MATERIALS AND METHODS}

Sampling of isolates. On 11 August 2010, samples of $P$. infestans were collected in a potato field with alternating rows of 'Perlo', 'Princess', 'Amadine', 'Superb', and 'King Edward VII'. The field, located in southeast Sweden $\left(55^{\circ} 23^{\prime} \mathrm{N}, 14^{\circ} 3^{\prime} \mathrm{E}\right)$, was heavily infested with hairy nightshade. Both potato and hairy nightshade showed typical symptoms of late blight. Twenty-five single-lesion leaves per host were randomly collected. All infected leaves were placed in petri dishes with water agar directly in the field. After sampling, the dishes were incubated at $16^{\circ} \mathrm{C}$ with $16 \mathrm{~h}$ of light and $8 \mathrm{~h}$ of darkness.

Aggressiveness tests. After 1 day of incubation, sporangia had formed on all the collected leaves. The sporangia were rinsed off and collected in distilled water according to Lehtinen et al. (24). The concentration of sporangia was adjusted to $10^{4}$ sporangia ml ${ }^{-1}$ and was kept at $4^{\circ} \mathrm{C}$ for $3 \mathrm{~h}$ to promote zoospore formation. Each sporangial suspension was considered as one isolate. After the sporangia were harvested, all leaves were dried and stored for genotyping of $P$. infestans.

To set up the aggressiveness test, potato and hairy nightshade leaves without symptoms of late blight were collected in fieldgrown plants not treated with fungicides. For potato, 'Sava', with medium resistance to late blight, was used. The two leaf types of hairy nightshade (entire or dentate leaf margins) (12) were present in the sampled field but only leaves with entire leaf margins were used in the aggressiveness tests. All leaves were placed on water agar in petri dishes and inoculated by depositing one 20 - $\mu$ l droplet of sporangial suspension on the abaxial side of the leaflet close to the middle vein. The inoculated leaves were incubated at $16^{\circ} \mathrm{C}$ with $16 \mathrm{~h}$ of light and $8 \mathrm{~h}$ of darkness. The two different hosts were inoculated with late blight isolates originating from potato or hairy nightshade. Five replicates were used for each isolatehost combination.

The proportion of infected leaves and the latency period, lesion growth rate, and number of sporangia produced per lesion were used to assess aggressiveness of the sampled $P$. infestans isolates. The proportion of infected leaves (of the five replicates) for each isolate was recorded. The length of the latency period was measured as the time from inoculation to the time when the first sporangia were observed. The leaves were checked for sporulating lesions twice daily using a dissection microscope. Two measurements of the diameter of each lesion were taken at right angles once a day. When the lesion reached the leaf margin, no further measurements were taken. The area of the lesions was calculated using the formula for an ellipse. The lesion growth rate was determined as the slope of the regression line by plotting lesion size against the day of measurement. The experiment was finalized 11 days after inoculation. To determine the number of sporangia produced per lesion, the leaves were transferred to Falcon tubes with $5 \mathrm{ml}$ of $70 \%$ ethanol after finalizing the tests. The tubes were stored at $4^{\circ} \mathrm{C}$. Each tube was vortexed for $10 \mathrm{~s}$, and the number of sporangia for each suspension was counted using a hemocytometer.
Sporulation was measured as number of sporangia per lesion because no correlation between the final lesion area and number of sporangia was found. When no sporangia formation was observed during incubation, latency periods were treated as the missing value. When neither lesions nor sporangia formation were observed (i.e., no infection), lesion growth rate and sporulation per lesion for that isolate-inoculated host combination were treated as missing values. When sporangia but no lesions were observed, lesion growth was set to zero.

Isolation and determination of mating type. A small amount of mycelium was taken from all leaves used in the aggressiveness tests and transferred to petri dishes containing a 1:1 mixture of rye (5) and pea medium (protocol from Corbière and Andrivon [6]) amended with ampicillin (Sigma) at $0.2 \mathrm{~g} \mathrm{liter}^{-1}$. After 1 week, small agar plugs with mycelium were transferred to new petri dishes with antibiotic free rye-pea medium. Conventional mating tests were carried out on rye-pea agar with known A1 and A2 reference isolates (25).

Genotype identification. Pieces of the dried leaves of potato and hairy nightshade from the aggressiveness tests were cut and placed in sterile 1.5-ml tubes together with glass beads and then homogenized with a Precellys preparation shaker (Bertin Technologies). DNA was extracted according to a cetyltrimethylammonium bromide-based protocol (16). The genotypes were characterized using a set of seven SSR markers: 4B and G11 (20) and D13, Pi04, Pi16, Pi63, and Pi70 (23). The forward primers 4B, Pi16, D13, and Pi04 were labeled with the fluorescent dye FAM (TAC Copenhagen) and G11, Pi70, and Pi63 were labeled with NED (Applied Biosystems). The polymerase chain reaction (PCR) was carried out in a total volume of $15 \mu \mathrm{l}$ containing 10 to $30 \mathrm{ng}$ of DNA, 0.6 U of DreamTaq DNA polymerase (Fermentas) and corresponding $1 \times$ DreamTaq Green Buffer containing $2 \mathrm{mM}$ $\mathrm{MgCl}_{2}$, and $0.3 \mu \mathrm{M}$ forward and reverse primers. For primers for locus $4 \mathrm{~B}, 4 \mathrm{mM} \mathrm{MgCl}_{2}$ was used. Different annealing temperatures and number of cycles were used for each primer pair. For $\mathrm{D} 13$, the lowest annealing temperature, $50^{\circ} \mathrm{C}$, and 33 cycles were used, and 33 cycles were also used for 4B, Pi16, Pi70, and Pi63. For $4 \mathrm{~B}$ and $\mathrm{Pi70}$, an annealing temperature of $58^{\circ} \mathrm{C}$ was used and, for Pi63, the annealing temperature was $60^{\circ} \mathrm{C}$. For the primers G11 and Pi04, only 30 cycles were used, with an annealing temperature of $62^{\circ} \mathrm{C}$. All PCR products were analyzed using a GeneAmp PCR System 2700 (Applied Biosystems). The amplified fluorescently labeled PCR products were analyzed at Uppsala Genome Center, Rudbeck Laboratory, Uppsala University with an ABI 3730xl DNA analyzer (Applied Biosystems). The fragment length of the alleles was determined with the software Genemarker (version 1.80; Softgenetics).

Statistical analyses. All statistical analyses concerning the aggressiveness tests were performed using SAS version 9.2. All analyses were based on the grouping from the cross-inoculation experiment (isolates from potato tested on potato, isolates from potato tested on nightshade, isolates from nightshade tested on potato, and isolates from nightshade tested on nightshade). Because the ability to infect a leaf (or not) is a binary outcome, analysis of these data was performed using logistic regression. The dependent variable was the number of successful infections, and the denominator was the number of leaves, usually five but sometimes three or four due to lost replicates. In this model, the logit of the proportion of infected leaves was related to isolate origin and inoculated host. The remaining dependent variables were considered to be continuous. The measurements from the individual leaves in each petri dish are pseudoreplicates; therefore, the effects of isolate origin and inoculated source, as well as their interactions, were determined using the methods proposed by Oyarzun et al. (28). A closer examination of the data revealed that the variance of the residuals from the analysis of some variables was not constant, which would give inaccurate $P$ values. $F$ tests, used to examine the stability of variance between isolates 
and between host plants, revealed several combinations where the variance was clearly not uniform. For this reason, pairwise testing via $t$ tests was done to analyze the differences between and within hosts. The $P$ values for these components of aggressiveness were calculated using individual variances. If differences in variance was found, Satterthwaite's approximation was used to estimate degrees of freedom.

Genotypic differentiation was assessed by counting the number of pairwise genetic differences between all isolates. This difference was used in a principal coordinate analysis (PCA) to visualize any clustering based on isolate origin. Genetic distance and PCA was calculated in GENALEX version 6.41 (29).

\section{RESULTS}

In the logistic regression examining the ability to infect the leaves, the independent variables "source of inoculum" and "inoculated host" plant were both significant $(P<0.05)$. The interaction between these two was not significant (Table 1). In a model without this interaction term, the odds of infection (both hosts combined) with isolates from hairy nightshade was 1.81 (95\% confidence interval $[\mathrm{CI}]=1.20$ to 2.74$)$ times greater than the odds for infection with isolates from potato. When comparing infection of the two hosts, the odds for hairy nightshade (all isolates combined) was only of $0.38(95 \% \mathrm{CI}=0.25$ to 0.57$)$ compared with potato. Although the interaction term was not statistically significant at $P=0.05$, examination of the results of a model with an interaction term tended to show higher odds of infection for isolates from hairy nightshade tested on hairy nightshade plants (data not shown). In other words, the nightshade isolates could more easily infect nightshade plants compared with the potato isolates but this difference was not seen on potato plants.

In the analysis of variance with the continuous dependent variables, a significant interaction (inoculated host-isolate origin) was not found in any of the measured aggressiveness components. The main effect of isolate origin was significant for sporulation. Inoculated host showed statistically significant main effects for latency period and sporulation (Table 2). Separate analyses of the two hosts showed that isolates from hairy nightshade had a significantly shorter latency period and a higher sporulation capacity compared with isolates from potato when inoculated on potato (Fig. 1). No significant differences in isolate origin for latency period and lesion growth could be found when the same isolate was used to inoculate nightshade leaves. As in potato, the sporulation capacity was higher in isolates originating from hairy nightshade than in the ones sampled from potato (Fig. 2).

The mating type test showed a ratio close to $1: 1$ between mating types $\mathrm{A} 1$ and $\mathrm{A} 2$ in both potato and hairy nightshade isolates. In the 50 isolates sampled, 39 isolates represented genotypes found only once (Table 3 ). However, no genotypic differ-

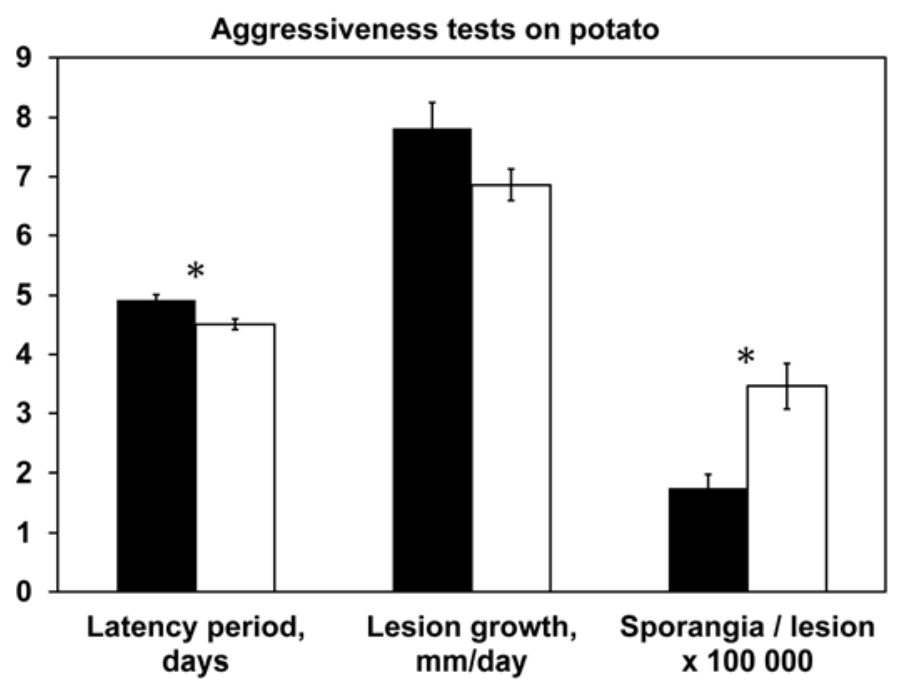

\section{- Isolates from potato $\quad$ lsolates from hairy nightshade}

Fig. 1. Aggressiveness tests performed on potato leaves. Bars represent mean latency period in days, lesion growth rate $(\mathrm{mm} / \mathrm{day})$, and sporangia per lesion for Phytophthora infestans isolates collected from hairy nightshade or potato. Vertical lines represent the standard errors and asterisks indicate significant differences between the isolate origin $(P<0.05)$.

TABLE 1. Analysis of deviance from a logistic regression model examining the effects of Phytophthora infestans isolate origin (potato or hairy nightshade) and inoculated host (potato or hairy nightshade) of the odds of infection

\begin{tabular}{lccrr}
\hline Source & Deviance & df & $\chi^{2}$ & \\
\hline Origin & 256.22 & 1 & 7.92 & 0.0049 \\
Inoculated host & 248.30 & 1 & 21.72 & $<0.0001$ \\
Origin $\times$ inoculated host & 223.58 & 1 & 3.00 & 0.0831 \\
\hline
\end{tabular}

TABLE 2. Analysis of variance and mean main effects from a factorial detached leaf assay examine the possible interaction of Phytophthora infestans isolate origin (potato or hairy nightshade) and inoculated host (potato or hairy nightshade) ${ }^{\mathrm{a}}$

\begin{tabular}{|c|c|c|c|c|c|c|c|c|c|c|}
\hline \multirow[b]{3}{*}{ Source } & \multirow[b]{3}{*}{$\mathrm{df}$} & \multirow[b]{3}{*}{ Mean square } & \multirow[b]{3}{*}{$F$ value } & \multirow[b]{3}{*}{$P>F$} & \multicolumn{6}{|c|}{ Main effect } \\
\hline & & & & & \multicolumn{3}{|c|}{ Isolate } & \multicolumn{3}{|c|}{ Inoculated } \\
\hline & & & & & $\mathrm{HN}$ & Potato & SD & $\mathrm{HN}$ & Potato & SD \\
\hline Isolate & 1 & 0.21 & 0.08 & 0.7766 & $\ldots$ & $\ldots$ & $\ldots$ & $\ldots$ & $\ldots$ & $\ldots$ \\
\hline Host & 1 & 244.26 & 96.21 & $<0.0001$ & $\ldots$ & $\ldots$ & $\ldots$ & $\ldots$ & $\ldots$ & $\ldots$ \\
\hline Isolate $\times$ host & 1 & 4.85 & 1.91 & 0.1753 & $\ldots$ & $\ldots$ & $\ldots$ & $\ldots$ & $\ldots$ & \\
\hline Growth rate & $\ldots$ & $\ldots$ & $\ldots$ & $\ldots$ & 6.8 & 7.79 & Yes & 7.15 & 7.35 & No \\
\hline Isolate $\times$ host & 1 & 1.61 & 0.12 & 0.7304 & $\ldots$ & $\ldots$ & $\ldots$ & $\ldots$ & $\ldots$ & $\ldots$ \\
\hline Sporangia/lesion & $\ldots$ & $\ldots$ & & & 307.8 & 159.18 & Yes & 196.91 & 259.44 & Yes \\
\hline Isolate & 1 & 954230 & 18.34 & 0.0001 & $\ldots$ & $\ldots$ & $\ldots$ & $\ldots$ & $\ldots$ & $\ldots$ \\
\hline Host & 1 & 563219 & 10.82 & 0.0022 & $\ldots$ & $\ldots$ & $\ldots$ & $\ldots$ & $\ldots$ & $\ldots$ \\
\hline Isolate $\times$ host & 1 & 52737 & 1.01 & 0.3206 & $\ldots$ & $\ldots$ & $\ldots$ & $\ldots$ & $\ldots$ & $\ldots$ \\
\hline
\end{tabular}

${ }^{a}$ Dependent variables examined were latency period in days (Latency), number of sporangia per lesion, and lesion growth rate ( $\mathrm{mm} /$ day); Isolate $=$ isolate origin, Host $=$ inoculated host, $\mathrm{HN}=$ hairy nightshade, and $\mathrm{SD}=$ significant difference . 
entiation in the isolates collected from the two hosts could be observed (Fig. 3).

\section{DISCUSSION}

This study indicates that presence of the alternative host $S$. physalifolium can increase the aggressiveness of $P$. infestans on potato. Compared with isolates sampled from potato, isolates from hairy nightshade showed a shorter latency period and higher sporulation when inoculated on potato. The lower odds of infection for hairy nightshade suggest that only parts of the P. infestans population in the sampled field were able to infect this host. This will mean that "weaker" isolates restricted to potato will be outcompeted by the isolates coming from nightshade. In this way, the dynamics of a late blight epidemic with a pathogen switching between sympatric hosts will filter the population toward an increased aggressiveness on potato crops.

The late blight epidemic in the sampled field probably started on potato because the potato crop would be established before the hairy nightshade. Our results suggest that it is easier for $P$. infestans to infect potato than hairy nightshade. Despite this, almost all of the hairy nightshade plants we observed in the sampling region were infected. The question is, why will the pathogen infect hairy nightshade at all? One explanation could be the structure of the local $P$. infestans population. In contrast to most other reports on aggressiveness in $P$. infestans, our study was done on a nonclonal population of the pathogen. Due to widespread sexual recombination $(3,32)$, P. infestans in Sweden is more genotypically diverse compared with most other studied areas. In a nonclonal population of a pathogen, sexual reproduction will give a higher probability of generating genotypes

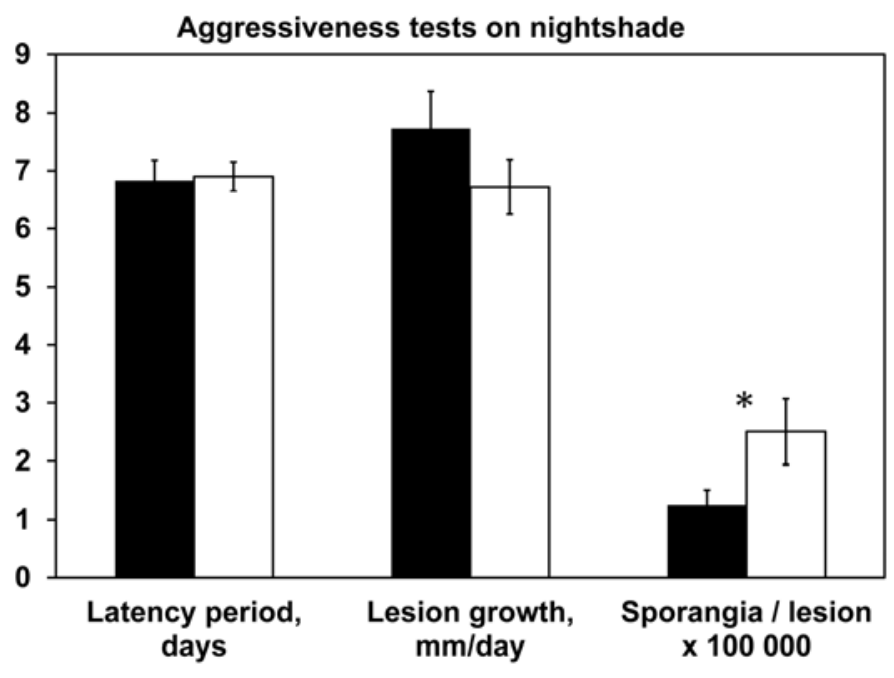

- Isolates from potato $\square$ Isolates from hairy nightshade

Fig. 2. Aggressiveness tests performed on hairy nightshade leaves. Bars represent mean latency period in days, lesion growth rate $(\mathrm{mm} / \mathrm{day})$, and sporangia per lesion for Phytophthora infestans isolates collected from hairy nightshade or potato. Vertical lines represent the standard errors and asterisks indicate significant differences between the isolate origin $(P<0.05)$.

TABLE 3. Distribution of mating type and genotype in Phytophthora infestans isolates collected from potato or hairy nightshade (HN)

\begin{tabular}{lcccc}
\hline & \multicolumn{3}{c}{ Mating type } \\
\cline { 2 - 4 } Isolate origin & $\mathrm{A} 1$ & $\mathrm{~A} 2$ & $\mathrm{NT}^{\mathrm{a}}$ & Genotypes $^{\mathrm{b}}$ \\
\hline Potato & 7 & 13 & 5 & 20 \\
HN & 13 & 10 & 2 & 19 \\
\hline
\end{tabular}

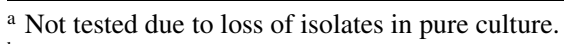

${ }^{\mathrm{b}}$ Number of simple-sequence repeat genotypes. able to infect new hosts. In the pathosystem $P$. infestans-potato, both pathogen and host are usually clonal. In some areas, like northern Europe, $P$. infestans is sexually recombining while potato is vegetatively propagated. However, in the case of $P$. infestans-hairy nightshade, both pathogen and host are sexually reproducing. This will result in an increased selection for more aggressive genotypes of the pathogen. Due to the short generation time of $P$. infestans, one single growing season could be sufficient to increase the aggressiveness of the pathogen population. At the same time, the hairy nightshade population will be selected toward increased resistance. In our study, the sampling was done in a late stage of the epidemic which could partly explain the differences found in the aggressiveness components for the two hosts.

As could be expected, a high genotypic variation was found among the isolates collected in this study. However, no genetic population differentiation in the isolates originating from the two hosts could be observed when analyzed using SSR markers. Other population studies have shown genetic differentiation of $P$. infestans on different hosts $(11,20,21,26)$. In our study, the $P$. infestans isolates were sampled from the two hosts in the same field. It can be assumed that, if the two hosts would have been in allopatry, the populations might have been more genetically differentiated.

Oospore formation of $P$. infestans has been reported in $S$. physalifolium (1). Oospores acting as a source of inoculum will increase genotypic variation and, thereby, the adaptability of the pathogen population. Oospore formation in both hosts indicates that reproductive barriers are unlikely, which will reduce the rate of host adaptation. In our results, the isolates from hairy nightshade showed a significantly higher sporulation capacity on both hosts, suggesting no host adaptation in the pathogen population. Isolates from potato tended to have a higher lesion growth rate compared with isolates from hairy nightshade, though this difference was not statistically significant. Overall, the results from our study show that the pathosystem potato-hairy nightshade- $P$. infestans differs from the usual pattern of higher aggressiveness of plant pathogens on their host of origin (17).

This study was done based on sampling of isolates of $P$. infestans in a single field. The results suggest that, in the investigated field, the presence of an alternative host increased the aggres-

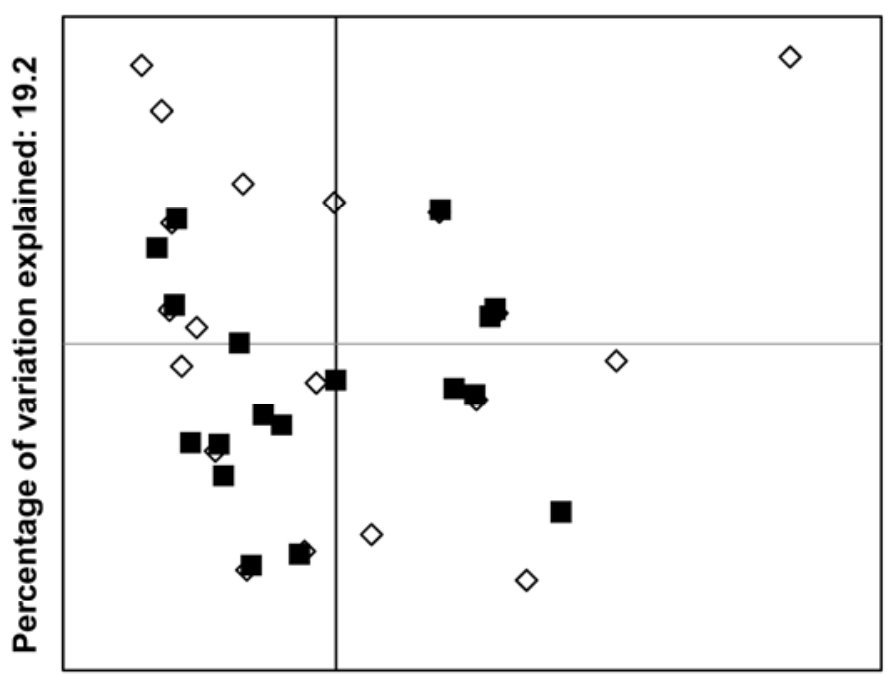

Percentage of variation explained: $\mathbf{5 4 . 3}$

\section{$\diamond$ Nightshade $\square$ Potato}

Fig. 3. Principal component analysis of multilocus genotypes of Phytophthora infestans isolates collected from hairy nightshade or potato generated from a genetic distance matrix to visualize any clustering based on isolate origin. 
siveness of the pathogen on potato. However, this only describes the situation when the two hosts are sympatric. For future research, it would be of interest to study whether the result would be the same when hairy nightshade is the only host present for $P$. infestans. Would several generations on an alternative and less susceptible host increase the aggressiveness even more?

In Swedish potato production, crop rotation is normally practiced to manage problems with soil borne pathogens. Hairy nightshade is locally both a serious weed and an alternative host to $P$. infestans. If infected, this alternative host will function as a reservoir for the pathogen by supporting oospore production. This fact will reduce or even eliminate the effect of crop rotation. Earlier epidemics, possibly caused by oospores, in combination with an increased aggressiveness will have serious consequences for the control of potato late blight.

\section{LITERATURE CITED}

1. Andersson, B., Johansson, M., and Jonsson, B. 2003. First report of Solanum physalifolium as a host plant for Phytophthora infestans in Sweden. Plant Dis. 87:1538-1538.

2. Andersson, B., Sandström, M., and Strömberg, A. 1998. Indications of soil borne inoculum of Phytophthora infestans. Potato Res. 41:305-310.

3. Brurberg, M. B., Elameen, A., Le, V. H., Naerstad, R., Hermansen, A., Lehtinen, A., Hannukkala, A., Nielsen, B., Hansen, J., Andersson, B., and Yuen, J. 2011. Genetic analysis of Phytophthora infestans populations in the Nordic countries reveals high genetic variability. Fungal Biol. 115:335-342.

4. Carlisle, D. J., Cooke, L. R., Watson, S., and Brown, A. E. 2002. Foliar aggressiveness of Northern Ireland isolates of Phytophthora infestans on detached leaflets of three potato cultivars. Plant Pathol. 51:424-434.

5. Caten, C. E., and Jinks, J. L. 1968. Spontaneous variability of single isolates of Phytophthora infestans. I. Cultural variation. Can. J. Bot. 46:329-348.

6. Cobière, R., and Andrivon, D. 2003. Preparation of a simple pea medium for culturing Phytophthora infestans. http://www.eucablight.org/ Eucablight.asp.

7. Cooke, L. R., Carlisle, D. J., Wilson, D. G., and Deahl, K. L. 2002. Natural occurrence of Phytophthora infestans on woody nightshade (Solanum dulcamara) in Ireland. Plant Pathol. 51:392-392.

8. Day, J., and Shattock, R. 1997. Aggressiveness and other factors relating to displacement of populations of Phytophthora infestans in England and Wales. Eur. J. Plant Pathol. 103:379-391.

9. Deahl, K. L., Shaw, D. S., and Cooke, L. R. 2004. Natural occurrence of Phytophthora infestans on black nightshade (Solanum nigrum) in Wales. Plant Dis. 88:771-771.

10. Edmonds, J. M. 1986. Biosystematics of Solanum sarrachoides Sendtner and $S$. physalifolium Rusby (S. nitidibaccatum Bitter). Bot. J. Linn. Soc. 92:1-38.

11. Erselius, L. J., Hohl, H. R., Ordonez, M. E., Oyarzun, P. J., Jarrin, F., Velasco, A., Ramon, M. P., and Forbes, G. A. 1999. Genetic diversity among isolates of Phytophthora infestans from various hosts in Ecuador. Pages 39-48 in: Impact on a Changing World. Program Report 19971998. International Potato Center, Lima, Peru.

12. Erwin, D. C., and Ribeiro, O. K. 1996. Phytophthora Diseases Worldwide. American Phytopathological Society Press, St. Paul, MN.

13. Flier, W. G., van den Bosch, G. B. M., and Turkensteen, L. J. 2003. Epidemiological importance of Solanum sisymbriifolium, S. nigrum and S. dulcamara as alternative hosts for Phytophthora infestans. Plant Pathol. 52:595-603.
14. Flier, W. G., and Turkensteen, L. 1999. Foliar aggressiveness of Phytophthora infestans in three potato growing regions in the Netherlands. Eur. J. Plant Pathol. 105:381-388.

15. Fontem, D. A., Olanya, O. M., and Njualem, B. F. 2004. Reaction of certain solanaceous and asteraceous plant species to inoculation with Phytophthora infestans in Cameroon. J. Phytopathol. 152:331-336.

16. Gardes, M., and Bruns, T. D. 1993. ITS primers with enhanced specificity for Basidiomycetes - application to the identification of mycorrhizae and rusts. Mol. Ecol. 2:113-118.

17. Garry, G., Forbes, G. A., Salas, A., Santa Cruz, M., Perez, W. G., and Nelson, R. J. 2005. Genetic diversity and host differentiation among isolates of Phytophthora infestans from cultivated potato and wild solanaceous hosts in Peru. Plant Pathol. 54:740-748.

18. Haverkort, A. J., Boonekamp, P. M., Hutten, R., Jacobsen, E., Lotz, L. A. P., Kessel, G. J. T., Visser, R. G. F., and Van der Vossen, E. A. G. 2008. Societal costs of late blight in potato and prospects of durable resistance through cisgenic modification. Potato Res. 51:47-57.

19. Hirst, J. M., and Stedman, O. J. 1960. The epidemiology of Phytophthora infestans II. The source of inoculum. Ann. Appl. Biol. 48:489-517.

20. Knapova, G., Tenzer, I., Gessler, C., and Gisi, U. 2001. Characterisation of Phytophthora infestans from potato and tomato with molecular markers. In: The 5th Congress of the European Foundation for Plant Pathology. Taormina, Italy.

21. Lebreton, L., and Andrivon, D. 1998. French isolates of Phytophthora infestans from potato and tomato differ in phenotype and genotype. Eur. J. Plant Pathol. 104:583-594.

22. Lebreton, L., Lucas, J. M., and Andrivon, D. 1999. Aggressiveness and competitive fitness of Phytophthora infestans isolates collected from potato and tomato in France. Phytopathology 89:679-686.

23. Lees, A. K., Wattier, R., Shaw, D. S., Sullivan, L., Williams, N. A., and Cooke, D. E. L. 2006. Novel microsatellite markers for the analysis of Phytophthora infestans populations. Plant Pathol. 55:311-319.

24. Lehtinen, A., Andersson, B., Le, V. H., Naerstad, R., Rastas, M., Ketoja, E., Hannukkala, A. O., Hermansen, A., Nielsen, B. J., Hansen, J. G., and Yuen, J. 2009. Aggressiveness of Phytophthora infestans on detached potato leaflets in four Nordic countries. Plant Pathol. 58:690-702.

25. Lehtinen, A., Hannukkala, A., Andersson, B., Hermansen, A., Le, V. H., Naerstad, R., Brurberg, M. B., Nielsen, B. J., Hansen, J. G., and Yuen, J. 2008. Phenotypic variation in Nordic populations of Phytophthora infestans in 2003. Plant Pathol. 57:227-234.

26. Möller, K., Dilger, M., Habermeyer, J., Zinkernagel, V., Flier, W. G., and Hausladen, H. 2009. Population studies on Phytophthora infestans on potatoes and tomatoes in southern Germany. Eur. J. Plant Pathol. 124:659-672.

27. Olanya, O. M., Lambert, D. H., and Plant, A. B. 2005. Occurrence and cross-infection of Phytophthora infestans on hairy nightshade (Solanum sarrachoides) and potato (Solanum tuberosum) in Maine. Can. J. Plant Pathol. 27:458-460.

28. Oyarzun, P. J., Pozo, A., Ordonez, M. E., Doucett, K., and Forbes, G. A. 1998. Host specificity of Phytophthora infestans on tomato and potato in Ecuador. Phytopathology 88:265-271.

29. Peakall, R., and Smouse, P. E. 2006. GENALEX 6: Genetic analysis in Excel. Population genetic software for teaching and research. Mol. Ecol. Notes 6:288-295.

30. Platt, H. W. 1999. Response of solanaceous cultivated plants and weed species to inoculation with A1 or A2 mating time strains of Phytophthora infestans. Can. J. Plant Pathol. 21:301-307.

31. Vanderplank, J. 1963. Plant Diseases: Epidemics and Control. Academic Press, New York.

32. Widmark, A.-K., Andersson, B., Cassel-Lundhagen, A., Sandstrom, M., and Yuen, J. E. 2007. Phytophthora infestans in a single field in southwest Sweden early in spring: Symptoms, spatial distribution and genotypic variation. Plant Pathol. 56:573-579. 